\title{
Cuidados ao recém-nascido e orientações às puérperas no alojamento conjunto
}

\author{
Newborn care and guidance to postpartum women in rooming-in \\ Atención y orientación del recién nacido para mujeres posparto en alojamiento conjunto
}

Recebido: 29/11/2021 | Revisado: 07/12/2021 | Aceito: 10/12/2021 | Publicado: 18/12/2021

\author{
Brenda Geovana Furlan \\ ORCID: https://orcid.org/0000-0003-1217-0587 \\ Universidade Estadual de Londrina, Brasil \\ E-mail: brendageovanafurlan@hotmail.com \\ Juliane Pagliari Araujo \\ ORCID: https://orcid.org/0000-0001-7821-6731 \\ Universidade Estadual de Londrina, Brasil \\ E-mail:juliane.pagliari@uel.br \\ Milena Torres Guilhem Lago \\ ORCID: https://orcid.org/0000-0002-7735-5905 \\ Universidade Estadual de Londrina, Brasil \\ E-mail: milena_mtg@hotmail.com \\ Keli Regiane Tomeleri da Fonseca Pinto \\ ORCID: https://orcid.org/0000-0003-1280-8421 \\ Universidade Estadual de Londrina, Brasil \\ E-mail: tomeleri@yahoo.com.br \\ Rosangela Aparecida Pimenta Ferrari \\ ORCID https://orcid.org/0000-0003-0157-7461 \\ Universidade Estadual de Londrina, Brasil \\ E-mail: ropimentaferrari@uel.br \\ Adriana Valongo Zani \\ ORCID https://orcid.org/0000-0002-6656-8155 \\ Universidade Estadual de Londrina, Brasil \\ E-mail: adrianazanienf@gmail.com
}

\begin{abstract}
Resumo
Objetivo: identificar os cuidados prestados ao recém-nascido e orientações às puérperas no alojamento conjunto. Método: estudo descritivo, transversal, realizado com 290 puérperas em duas maternidades públicas, vinculadas ao Programa Rede Mãe Paranaense, no período de agosto a dezembro de 2017. As variáveis foram analisadas de modo descritivo, considerando frequência simples e percentual. Resultados: a administração de vacina contra Hepatite B, administração de vitamina $\mathrm{K}$, teste do pezinho e do coraçãozinho foram realizados em $100 \%$ dos recém-nascidos, de ambas as maternidades. As orientações sobre vantagens do aleitamento materno, estratégias para auxiliar a amamentar, à não utilização de chupetas/mamadeiras, apoio ao executar primeiros cuidados com o bebê são ações que precisam de maior engajamento por parte da equipe multiprofissional, pois apesar de serem realizadas, ainda não atinge a totalidade das puérperas. Conclusão: Os cuidados ao recém-nascido como administração de vitamina K, realização dos testes de triagem neonatal já são práticas realizadas na totalidade nas maternidades estudadas. No entanto, com relação ao apoio e orientações para higiene do recém-nascido e aleitamento materno, apesar de consolidadas, ainda apresentam algumas fragilidades.
\end{abstract}

Palavras-chave: Alojamento conjunto; Recém-nascido; Cuidados de enfermagem; Saúde da criança; Programas de saúde.

\begin{abstract}
Objective: to identify the care provided to the newborn and guidelines for postpartum women in rooming-in. Methods: descriptive, cross-sectional study, carried out with 290 mothers in two public maternity hospitals, linked to the Rede Mãe Paranaense Program, from August to December 2017. The variables were analyzed descriptively, considering simple frequency and percentage. Results: administration of vaccine against Hepatitis B, administration of vitamin K, newborn and heart tests were performed in $100 \%$ of newborns, from both maternity hospitals. Guidance on the advantages of breastfeeding, strategies to help breastfeeding, not using pacifiers/bottles, support when performing first care with the baby are actions that need greater engagement by the multidisciplinary team, as despite being carried out, they are still it does not reach all puerperal women. Conclusion: Newborn care such as administration of vitamin $\mathrm{K}$, carrying out neonatal screening tests are already practices performed almost entirely in the studied maternity hospitals. However, with regard to support and guidance for newborn hygiene and breastfeeding, despite being consolidated, they still have some weaknesses.
\end{abstract}

Keywords: Rooming-in care; Newborn; Nursing care; Child health; Health programs. 


\begin{abstract}
Resumen
Objetivo: identificar la atención brindada al recién nacido y pautas para la puérpera en alojamiento conjunto. Métodos: estudio descriptivo, transversal, realizado con 290 madres en dos maternidades públicas, vinculadas al Programa Rede Mãe Paranaense, de agosto a diciembre de 2017. Las variables se analizaron de forma descriptiva, considerando frecuencia simple y porcentaje. Resultados: se realizó administración de vacuna contra Hepatitis B, administración de vitamina $\mathrm{K}$, pruebas neonatales y cardíacas en el 100\% de los recién nacidos de ambas maternidades. Orientación sobre las ventajas de la lactancia materna, estrategias para ayudar a la lactancia materna, no usar chupetes / biberones, apoyo en la realización de los primeros cuidados con el bebé son acciones que necesitan un mayor compromiso por parte del equipo multidisciplinario, ya que a pesar de estar realizadas, aún no se alcanza. todas las puérperas. Conclusión: La atención al recién nacido como la administración de vitamina K, la realización de pruebas de cribado neonatal son prácticas realizadas casi en su totalidad en las maternidades estudiadas. Sin embargo, en cuanto al apoyo y orientación para la higiene del recién nacido y la lactancia materna, a pesar de estar consolidados, aún presentan algunas debilidades.
\end{abstract}

Palabras clave: Alojamiento conjunto; Recién nacido; Atención de enfermería; Salud del niño; Programas de salud.

\title{
1. Introdução
}

A reorganização das políticas públicas de saúde no Brasil, voltadas à população materno-infantil, perfaz longa caminhada, partindo de reflexões que denotavam lacunas no modo de atenção vigente (Araújo et al., 2014). Nesse contexto, a criação da Rede Cegonha visava fortalecer a assistência materno-infantil (Souza \& Santos, 2020).

Em 2012, no estado do Paraná, ocorreu a implantação do Programa Rede Mãe Paranaense (Santos et al., 2020), fundamentado no marco conceitual das Redes de Atenção à Saúde, como melhoria de acesso e qualidade da assistência materno-infantil. A estruturação das Redes de Atenção à Saúde baseia-se no fortalecimento e organização da Atenção Primária à Saúde como base na captação precoce da gestante, seu acompanhamento, e da criança até um ano de vida (Gomes et al., 2019; Paraná, 2018).

A Rede Mãe Paranaense tem como missão garantir o acesso e atenção, promovendo o cuidado seguro e de qualidade na gestação, parto e puerpério às crianças menores de um ano de idade (Gomes et al., 2019; Paraná, 2018).

Assim, integrando essa política de saúde, tem-se o alojamento conjunto (AC), o qual caracteriza-se por manter a puérpera e o recém-nascido juntos durante todo o período, desde o nascimento até a alta hospitalar. O objetivo está relacionado com o cuidado direto e integral entre mãe e filho, o favorecimento do vínculo entre os familiares, e com enfoque para as orientações e educação em saúde, realizadas e orientadas pela equipe de enfermagem. Além disso, o AC proporciona o incentivo e manutenção do aleitamento materno exclusivo e a redução da mortalidade neonatal (Costa \& Brito, 2016; Silva et al., 2018; Mercado et al., 2017).

No AC o enfermeiro tem um importante papel, tanto no atendimento da puérpera quanto do recém-nascido, pois ele realiza a sistematização da assistência, com prescrições de enfermagem, além de exercer ações educativas, como incentivo ao aleitamento materno, cuidados com o recém-nascido e esclarecimento de dúvidas das puérperas e seus familiares (Costa \& Brito, 2016; Mercado et al., 2017).

É importante reforçar que o intuito da educação em saúde no AC, não é o de impor o conhecimento, nem o de sobrecarregar a puérpera com tantas informações e novas adaptações (Strefling et al., 2017). Nesse sentido, o profissional deve tornar esse momento, um momento de troca de conhecimentos buscando realizar as orientações o mais próximo possível da realidade de cada puérpera, com incentivo positivo para realizar o autocuidado e prestar cuidados ao seu filho (Machado et al., 2021).

Considerando a importância do cuidado prestado ao recém-nascido no AC, com o objetivo de proporcionar fortalecimento de vínculo, manutenção do aleitamento materno e empoderamento da família para o cuidado do filho no domicílio, buscou-se responder ao questionamento: As maternidades públicas, integrantes do Programa Rede Mãe Paranaense, realizam os cuidados preconizados pelo programa na unidade de alojamento conjunto? 
Assim, o objetivo do presente estudo foi identificar os cuidados prestados ao recém-nascido e orientações às puérperas no alojamento conjunto.

\section{Método}

Trata-se de um estudo descritivo, alinhado a uma coorte prospectiva, o qual foi realizado em duas maternidades públicas, localizadas em um município na região norte do estado do Paraná, denominados neste estudo como maternidade A e B. A maternidade A é uma instituição municipal considerada modelo de cuidado às gestantes de risco habitual e intermediário, e que realiza em média 4.300 partos/ano, aproximadamente 360 partos/mês. A maternidade B está localizada em um hospital universitário, estadual, considerado centro regional de referência e modelo de cuidado a gestantes de alto risco, e, realiza em torno de 1983 parto/ano, ou seja, 165 partos/mês.

Participaram, do presente estudo, 290 puérperas, sendo que 227 receberam assistência ao parto na maternidade A e 63 na maternidade B. Para definição da amostra, considerou-se o número de partos do ano de 2016, com acréscimo de 20,0\%. Como critérios de inclusão, as mulheres deveriam ter realizado parto nas maternidades A e B entre agosto a dezembro de 2017 e que aceitaram participar do estudo. Foram excluídas as mulheres menores de 18 anos, desacompanhadas de um responsável, mães de natimortos ou de bebê que teve como desfecho: encaminhamento para unidade de terapia intensiva neonatal ou óbito nas primeiras horas de vida.

A coleta de dados ocorreu no período de agosto a dezembro de 2017, e foi realizada por discentes de graduação e pósgraduação em enfermagem, previamente treinados. Utilizou-se um instrumento próprio, elaborado para a presente pesquisa, e validado após realização de teste piloto. O questionário foi aplicado após as participantes lerem e assinarem o termo de consentimento livre e esclarecido. $\mathrm{O}$ local do encontro aconteceu em um espaço privado e reservado, na própria maternidade. Esse instrumento foi composto por dados sociodemográficos e clínicos, baseados em elementos da assistência referentes aos objetivos e pressupostos do Programa Rede Mãe Paranaense, o qual preconiza a redução da mortalidade materno-infantil e a garantia do funcionamento da rede para esse grupo, em todo o Paraná, no que se refere à assistência ao recém-nascido na unidade de alojamento conjunto (Paraná, 2018).

Utilizou-se, também, um instrumento para a busca das informações em documentos como prontuários do período de internação na maternidade. As variáveis analisadas, com relação aos dados sociodemográficos, foram: idade, situação conjugal, escolaridade, número de filhos e condição de ocupação, e as variáveis relacionadas à assistência ao recém-nascido (administração de vacina BCG, hepatite B, e vitamina K, administração de colírio de nitrato de prata, testes do pezinho, olhinho, coraçãozinho, orelhinha); orientações e apoio aos cuidados do recém-nascido (primeiros cuidados, banho, higiene perineal e troca de fraldas, curativo no coto umbilical, eliminação vesical e intestinal) e sobre orientações com relação à amamentação (vantagens do aleitamento materno, avaliação das mamadas, estratégias para auxiliar nas dificuldades para amamentar, pega correta, justificativas e orientações referentes à não utilização de chupetas e mamadeiras, como oferecer leite materno em copo nas situações de dificuldades de amamentação e/ou necessidade de complemento e uso de complemento pelo recém-nascido).

Usou-se a planilha eletrônica Microsoft Office Excel ${ }^{\circledR}$ para a construção do banco de dados. Estes foram inseridos por dupla digitação, seguida de validação para corrigir possíveis erros na introdução das informaçães, com posterior exportação para o programa R versão 64.4. Utilizou-se da estatística descritiva considerando frequência simples e percentual.

O presente estudo atendeu às questões éticas e legais vinculadas à pesquisa com seres humanos, analisada e aprovada pelo Comitê de Ética da Universidade vinculada ao estudo, sob CAAE: 67574517.1.1001.5231, aprovado sob o número 2.053.304/2017. 


\section{Resultados}

A análise das características demográficas da população estudada apontou que a maioria das puérperas tinham entre 19 e 36 anos, possuíam companheiro, e escolaridade de 8 a 11 anos. Em relação à via de nascimento ocorreu a predominância de parto vaginal na maternidade A, enquanto na maternidade B houve predomínio de parto cesárea (Tabela 1).

Tabela 1 - Caracterização sociodemográfica de puérperas atendidas em duas maternidades públicas. Brasil, 2017 (n=290).

\begin{tabular}{|c|c|c|}
\hline \multirow[t]{2}{*}{ Dados socioeconômicos } & $\begin{array}{c}\text { Maternidade A } \\
(\mathbf{n}=\mathbf{2 2 7})\end{array}$ & $\begin{array}{c}\text { Maternidade B } \\
(n=63)\end{array}$ \\
\hline & $\mathrm{n}(\%)$ & $\mathrm{n}(\%)$ \\
\hline \multicolumn{3}{|l|}{ Idade (em anos) } \\
\hline Até 18 & $33(14,5)$ & $3(4,7)$ \\
\hline 19 a 36 & $183(80,6)$ & $50(79,4)$ \\
\hline$>37$ & $11(4,9)$ & $10(15,9)$ \\
\hline \multicolumn{3}{|l|}{ Situação Conjugal } \\
\hline Com companheiro & $199(87,7)$ & $58(92,1)$ \\
\hline Sem companheiro & $28(12,3)$ & $5(7,9)$ \\
\hline \multicolumn{3}{|l|}{ Escolaridade (anos) } \\
\hline Baixa $(<7)$ & $74(32,6)$ & $20(31,7)$ \\
\hline Média (8 a 11) & $121(53,3)$ & $37(58,7)$ \\
\hline Alta $(>12)$ & $32(14,1)$ & $6(9,6)$ \\
\hline \multicolumn{3}{|l|}{ Número de filhos } \\
\hline 1 & $104(45,8)$ & $27(42,9)$ \\
\hline 2 & $73(32,2)$ & $22(35,0)$ \\
\hline 3 & $38(16,7)$ & $8(12,6)$ \\
\hline$>4$ & $12(5,3)$ & $6(9,5)$ \\
\hline \multicolumn{3}{|l|}{ Condição de ocupação } \\
\hline Remunerada & $90(39,6)$ & $28(44,4)$ \\
\hline Não remunerada & $137(60,4)$ & $35(55,6)$ \\
\hline \multicolumn{3}{|l|}{ Via de nascimento } \\
\hline Vaginal & $137(60,4)$ & $22(34,9)$ \\
\hline Cesárea & $90(39,6)$ & $41(65,1)$ \\
\hline
\end{tabular}

Fonte: Autores.

A análise das variáveis relacionadas aos procedimentos realizados com o recém-nascido, como administração de vacina contra Hepatite $\mathrm{B}$, administração de vitamina $\mathrm{K}$, teste do pezinho e do coraçãozinho, evidenciou que esses procedimentos foram realizados em 100,0\% dos recém-nascidos, nas duas maternidades. Apenas em um bebê não foi realizado teste do olhinho (maternidade A), pois o mesmo apresentava edema palpebral e foi encaminhado para serviço especializado. $\mathrm{O}$ teste da orelhinha foi realizado em $99,1 \%$ dos recém-nascidos da maternidade A e 100\% da maternidade B.

Com relação à vacina $\mathrm{BCG}$, a maternidade $\mathrm{B}$ não realiza, e encaminha todos os recém-nascidos para realização na atenção primária; já na maternidade A, 100\% dos recém-nascidos receberam essa vacina. Em relação à aplicação do colírio de nitrato de prata $1 \%$, para a profilaxia da oftalmia gonocócica, o qual deve ser realizado na primeira hora após o nascimento, os resultados apontam que, na maternidade A, 97,4\% dos recém-nascidos não receberam o colírio, tendo como justificativa a falta do medicamento na maternidade. Já na maternidade B, $61,9 \%$ não receberam o colírio, com a justificativa de que estes bebês nasceram por parto cesárea.

A Tabela 2 apresenta dados referentes às orientações para as puérperas relacionadas ao cuidado ao recém-nascido no alojamento conjunto. De modo geral, as orientações relacionadas aos primeiros cuidados ao recém-nascido, tais como banho, higiene perineal, troca de fraldas e curativo do coto umbilical em ambas as maternidades foi verificado que os profissionais realizaram estas orientações. 
Tabela 2 - Distribuição das orientações para as puérperas relacionadas ao cuidado ao recém-nascido no alojamento conjunto de duas maternidades públicas. Brasil, 2017. (n=290)

\begin{tabular}{lcc}
\hline Variáveis & $\begin{array}{c}\text { Maternidade A } \\
(\mathbf{n = 2 2 7})\end{array}$ & $\begin{array}{c}\text { Maternidade B } \\
(\mathbf{n = 6 3})\end{array}$ \\
\cline { 2 - 3 } & $\mathbf{n}(\mathbf{\%})$ & $\mathbf{n}(\mathbf{\%})$ \\
\hline Apoio ao executar primeiros cuidados & $211(92,9)$ & $51(81,0)$ \\
Sim & $16(7,1)$ & $12(19,0)$ \\
Não & & $40(63,5)$ \\
Apoio para a realização do banho & $200(88,1)$ & $23(36,5)$ \\
$\quad$ Sim & $27(11,9)$ & $44(69,9)$ \\
Não & & $19(30,1)$ \\
Apoio na execução do curativo do coto & $215(94,7)$ & $33(52,4)$ \\
Sim & $12(5,3)$ & $30(47,6)$ \\
Não & & $39(62,0)$ \\
Apoio na realização de higiene perineal e troca de fraldas & $177(78,0)$ & $24(38,0)$ \\
Sim & $50(22,0)$ & \\
Não & & $38(60,3)$ \\
Orientação sobre eliminação vesical & $182(80,2)$ & $25(39,7)$ \\
Sim & $45(19,8)$ & \\
Não & & $178(78,4)$ \\
Orientações sobre eliminação intestinal & $49(21,6)$ & \\
Sim & & \\
Não &
\end{tabular}

Fonte: Autores.

Com relação às orientações relacionadas à amamentação, a maioria das puérperas recebeu informações relacionadas às vantagens do aleitamento materno, assim como, tiveram avaliação da mamada do recém-nascido por um profissional, e receberam informações sobre estratégias para auxiliar nas dificuldades para amamentar. A maioria dos recém-nascidos não necessitou de complemento de leite durante o período de hospitalização, conforme Tabela 3. 
Tabela 3 - Distribuição das orientações referentes à amamentação, para puérperas, de duas maternidades públicas. Brasil, 2017. $(n=290)$.

\begin{tabular}{|c|c|c|}
\hline \multirow[t]{2}{*}{ Variáveis } & $\begin{array}{c}\text { Maternidade A } \\
(\mathbf{n}=\mathbf{2 2 7})\end{array}$ & $\begin{array}{c}\text { Maternidade B } \\
(n=63)\end{array}$ \\
\hline & $\mathrm{n}(\%)$ & $\mathrm{n}(\%)$ \\
\hline \multicolumn{3}{|c|}{ Vantagens do aleitamento materno } \\
\hline & & $34(54,0)$ \\
\hline Sim & $214(94,3)$ & $29(46,0)$ \\
\hline Não & $13(5,7)$ & \\
\hline \multicolumn{3}{|c|}{ Avaliação da mamada } \\
\hline Sim & $214(94,3)$ & $61(96,8)$ \\
\hline Não & $13(5,7)$ & $2(3,2)$ \\
\hline \multicolumn{3}{|c|}{ Estratégias para auxiliar na amamentação } \\
\hline Sim & $205(90,3)$ & $46(73,1)$ \\
\hline Não & $22(9,7)$ & $17(26,9)$ \\
\hline \multicolumn{3}{|c|}{$\begin{array}{l}\text { Orientações sobre pega correta, aleitamento materno em livre } \\
\text { demanda }\end{array}$} \\
\hline Sim & $168(74,0)$ & $61(96,8)$ \\
\hline Não & $59(26,0)$ & $2(3,2)$ \\
\hline \multicolumn{3}{|c|}{ Estratégias para auxiliar na amamentação } \\
\hline Sim & $151(66,6)$ & $20(31,8)$ \\
\hline Não & $76(33,4)$ & $43(68,2)$ \\
\hline \multicolumn{3}{|c|}{ Oferecer leite materno em copo (dificuldades de amamentação) } \\
\hline Sim & $49(21,6)$ & $13(20,7)$ \\
\hline Não & $178(78,4)$ & $50(79,3)$ \\
\hline \multicolumn{3}{|c|}{ Necessidade de utilização de complemento } \\
\hline Sim & $24(10,6)$ & $21(33,3)$ \\
\hline Não & $203(89,4)$ & $42(66,7)$ \\
\hline
\end{tabular}

Fonte: Autores.

\section{Discussão}

Considera-se que os cuidados apreendidos pelas mães no período de hospitalização no alojamento conjunto, proporciona uma experiência prazerosa para a mãe, promove a saúde por meio de práticas de cuidados básicos ao recémnascido como higiene e amamentação, e possibilita maior interação entre o binômio mãe-filho.

Pôde-se evidenciar que as políticas públicas voltadas à saúde infantil, baseadas nas diretrizes do Programa Rede Mãe Paranaense, são realizadas nas maternidades pesquisadas. No entanto, algumas ações, como orientações para as puérperas, precisam de maior engajamento por parte da equipe multiprofissional. $\mathrm{O}$ atendimento baseado na integralidade e equidade da assistência (Carnut, 2017), conforme as diretrizes do Sistema Único de Saúde, bem como, as políticas públicas de saúde direcionadas à saúde da criança mostra que as boas práticas nos cuidados refletem nas condições de saúde dos recém-nascidos.

Nesse sentido, a implementação das redes de atenção à saúde da mulher e da criança são primordiais para fortalecer sua autonomia e contribuir para a redução de riscos (Pereira Diaz et al., 2018). Essa premissa vem ao encontro do Programa Rede Mãe Paranaense que visa à Atenção Primária de qualidade, resolutiva e ordenadora do cuidado, com ações envolvendo pré-natal e puerpério, acompanhamento do crescimento e desenvolvimento das crianças, em especial, no seu primeiro ano de vida (Paraná, 2018).

Assim, faz-se necessário que os profissionais de saúde estejam habilitados para a construção e estabelecimento de vínculo, segurança e apoio à mulher e sua família (Silva et al., 2017), bem como, desenvolvam habilidades técnico-científicas para o atendimento ao recém-nascido nos primeiros dias de vida. Essas habilidades envolvem realizar o cuidado e orientar a mãe com relação aos cuidados com o recém-nascido. 
O Programa Rede Mãe Paranaense com foco na educação permanente da equipe multiprofissional apoia e incentiva o desenvolvimento dessas ações (Paraná, 2018).

No presente estudo foram identificados que, com relação aos testes de triagem neonatal, sendo estes, teste do pezinho, orelhinha, olhinho e coraçãozinho, estes são realizados nos recém-nascidos em ambas as maternidades, o que qualifica o cuidado nestes serviços. Ressalta-se que, nos casos de resultados desses testes alterados, o recém-nascido é encaminhado para serviço especializado como preconizado não apenas pelas diretrizes estaduais, mas também nacionais (Carvalho Tavares et al., 2020).

O acolhimento do recém-nascido e da família, pelos profissionais de saúde, é fundamental para a criação de vínculo, a partir de ações e condutas que expressem interesse, disponibilidade e respeito, contribuindo, assim, na redução de estresse, medos e angústias relacionados ao nascimento (Silva et al., 2017).

As orientações fornecidas para a mãe, na unidade de alojamento conjunto, são importantes para o desenvolvimento da autonomia e, deste modo, possam realizar os cuidados ao recém-nascido integralmente (higienização, cuidados com o coto umbilical, alimentação, entre outros) e, assim, prevenir possíveis infecções (Mercado et al.,).

Devido à imaturidade fisiológica e morfológica, o recém-nascido necessita de cuidado integral voltado para as suas particularidades de adaptações extrauterinas (Lima et al., 2020).

$\mathrm{O}$ apoio, da equipe multiprofissional, com relação aos cuidados ao recém-nascido, no que se refere ao apoio com relação ao banho, higiene e troca de fraldas, curativo do coto umbilical e cuidados em geral, deve-se estender tanto à primípara, quanto à multigesta. No presente estudo, muitas das justificativas para o não acompanhamento ou apoio à puérpera na realização de tais cuidados, consistem, justamente, no pressuposto de que esta já possuiria conhecimento, pelo fato de já ter outros filhos. Os achados se opõem a um estudo que demonstrou que todas as puérperas (100,0\%) foram orientadas quanto à higiene perineal do recém-nascido, assim como quanto à troca de fraldas (Mercado et al., 2017).

A enfermagem tem o papel de educar e orientar, para que as mulheres adquiram independência e autonomia nos cuidados do recém-nascido, tornando uma ferramenta indispensável para o planejamento da alta (Rogerio et al., 2020).

Com relação às orientações relacionadas ao aleitamento materno, sabe-se que além do vínculo afetivo do binômio, o aleitamento materno está relacionado à imunoproteção do bebê, sendo fundamental o papel do enfermeiro no processo de orientação. A prática do aleitamento materno traz vários benefícios para a criança, como: o ganho de peso mais rápido, prevenção contra icterícia e defesa contra infecções. As orientações sobre amamentação têm um papel fundamental no sucesso do aleitamento materno, pois são esclarecidas dúvidas referentes à pega correta, posicionamento, mitos e verdades, vantagens e desvantagens (Coca et al., 2020).

$\mathrm{O}$ incentivo à prática do aleitamento materno, por meio de orientações e apoio, proporciona redução do desmame precoce, bem como a utilização de mamadeiras e chupetas pode prejudicar significativamente este momento (Batista et al., 2017).

As maternidades participantes do estudo possuem o título de "Hospital Amigo da Criança", o qual incentiva a amamentação, por meio da promoção, proteção e apoio ao aleitamento materno (Margotti \& Margotti, 2017), além de estarem inseridas no Programa Rede Mãe Paranaense que, também, preconiza apoiar, promover e manter ações para o sucesso do aleitamento materno, com adoção dos "Dez Passos para o Sucesso do Aleitamento Materno" do Ministério da Saúde (Paraná, 2018).

No entanto, nem todas as mães receberam informações sobre vantagens do aleitamento materno, estratégias para auxiliar nas dificuldades para amamentar, informações de como manter a lactação nas situações em que o bebê não esteja sugando, pega correta, justificativas e orientações referentes a não utilização de chupetas e mamadeiras e necessidade de complemento. Vale ressaltar, a exemplo de outro estudo realizado no estado do Paraná, que ocorreu um número significativo 
de orientações consideradas adequadas pelas puérperas em relação ao aleitamento materno na unidade de alojamento conjunto (Genovesi et al., 2020).

Em estudo realizado no Rio Grande do Sul, verificou-se que as puérperas entrevistadas manifestaram ter recebido informações referentes aos cuidados com o bebê, como também, as orientações para elas (Strefling et al., 2017). Destaca-se, ainda, que o período em que o binômio se encontra no alojamento conjunto é de suma importância para a prática da amamentação, pois o início de tal prática é que favorece na avaliação de possíveis intervenções, conforme as necessidades apresentadas por cada mulher, bem como, é o momento propício para estimular e auxiliar nas dificuldades e dúvidas, além de contribuir para a sua confiança e autoestima (Mercado et al., 2017; Rogerio et al., 2020).

Salienta-se que, além da importância das políticas públicas vigentes, como a implantação de programas de atenção à saúde da criança, é necessário implementar capacitação e atualização profissional, somando-se a isso a mudança na formação dos profissionais de saúde (Silva et al., 2017) que atuam na atenção à saúde materno infantil.

Tem-se como limitação do presente estudo, a investigação de duas maternidades públicas, sendo necessário ampliar os estudos para outras regiões do estado do Paraná, bem como, para instituições privadas e/ou filantrópicas.

\section{Conclusão}

Os cuidados ao recém-nascido como administração de vitamina $\mathrm{K}$, vacina contra hepatite $\mathrm{B}$ e realização dos testes de triagem neonatal já são práticas realizadas quase a totalidade nas maternidades estudadas. No entanto, com relação ao apoio e orientações para higiene do recém-nascido e aleitamento materno, apesar de consolidadas, ainda apresentam algumas fragilidades. Diante desse contexto é necessário aprimorar a capacitação dos profissionais atuantes nas maternidades, com vistas ao pleno alcance dos objetivos do alojamento conjunto obstétrico.

Tem-se que a unidade de alojamento conjunto obstétrico é um local de atendimento com diferencial na relação entre a equipe de enfermagem e a puérpera, pois, os profissionais se colocam disponíveis para ajudá-la a vivenciar a experiência materna. Apresenta também, como uma de suas vantagens, as condições para a equipe de enfermagem promover demonstrações práticas dos cuidados indispensáveis ao recém-nascido.

\section{Referências}

Araújo, J. P., Silva, R. M. M., Collet, N., Neves, E. T., Toso, B. R. O. G., \& Viera, C. S. (2014). História da saúde da criança: conquistas, políticas e perspectivas. Revista Brasileira de Enfermagem [online]. 67; 6 https://doi.org/10.1590/0034-7167.2014670620.

Batista, C. L. C., Ribeiro, V. S., \& Nascimento, M. D. S. B. (2017). Influence of the use of artificial nipples and baby bottles in breastfeeding. J. Health Biol Sci. 5(2):184-91. http://dx.doi.org/10.12662/2317-3076jhbs.v5i2.1153.p184-191.2017

Carnut, L. (2017). Care, integrality and primary care: essential articulation to reflect on the health sector in Brazil. Saúde Debate. 41(115), 1177-86. http://dx.doi.org/10.1590/0103-1104201711515

Carvalho, B. M., Tavares, W. R., Vicente, J. B., Sanguino, G. Z., Leite, A. M., \& Furtado, M. C. D. C. (2020) Early access to biological neonatal screening: coordination among child care action programs. Rev Latino-Am Enfermagem. 28:1-7. http://dx.doi.org/ 10.1590/1518-8345.2938.3266

Coca, K. P., Pinto, V. L., Wesphal, F., Mania, P. N. A., \& Abrão, A. C. F. V. (2018). Bundle of measures to support intrahospital exclusive breastfeeding: evidence of systematic reviews. Rev Paul Pediatr. 36(2):214-20. https://doi.org/10.1590/1984-0462/;2018;36;2;00002

Costa, P. F., \& Brito, R. S. (2016). Guidance given to puerpera women at roomingin: an integrative review of the literature. Espaço Saúde. 17(2), 237-45. https://doi.org/10.22421/15177130-2016v17n2p237

Cruz, N. A. C. V. da, Reducino, L. M., Probst, L. F., Guerra, L. M., Ambrosano, G. M. B., Cortellazzi, K. L., \& et al. (2018). Association between type of breastfeeding of newborns at hospital discharge and at six months of age. Cad Saúde Colet. 26 (2):117-24. http://dx.doi.org/10.1590/1414-462X201800020349 
Genovesi, F. F., Canario, M. A. S. S., Godoy, C. B., Maciel, S. M., Cardelli, A. A. M., \& Ferrari, R. A. P. (2020) Maternal and child health care: adequacy index in public health services asistencia a la salud materno-infantil: índice de adecuación en servicios públicos de salud. Rev Bras Enferm. 73(Suppl4):e20170757. http://dx.doi.org/10.1590/0034-7167-2017-0757

Gomes, M. N. A., Santos, L. K. O., Matos, M. A. B., Lopes, P. R. R., Chomatas, E. R. B., Barra, R. P., \& et al. (2019). Nota técnica para organização da rede de atenção à saúde com foco na atenção primária à saúde e na atenção ambulatorial especializada - saúde da mulher na gestação, parto e puerpério. / Sociedade Beneficente Israelita Brasileira Albert Einstein. São Paulo: Hospital Israelita Albert Einstein: Ministério da Saúde.

Lima, R. O., Estevam, L. D., Leite, F. M., Almeida, M. V., Amorim, M. H. C. \& Bringuente, M. E. O. (2020). Intervenção de enfermagem-primeiro banho do recém-nascido: estudo randomizado sobre o comportamento neonatal. Acta Paul Enferm.33. http://dx.doi.org/10.37689/ acta-ape/2020AO0031

Machado, L. B., Andres, S. C., \& Moreschi, C. (2021). A atuação do enfermeiro no Alojamento Conjunto na promoção do aleitamento materno. Research, Society and Development, 10(1), e57410112266. https://doi.org/10.33448/rsd-v10i1.12266

Margotti, E., \& Margotti, W. (2017). Factors related to Exclusive Breastfeeding in Babies born in a child-friendly hospital in a capital of Northern Brazil. Saúde Debate. 41(114):860-71. https://doi.org/10.1590/0103-1104201711415

Mercado, N. C., Souza, G. D. S., Silva, M. M. J., \& Anseloni, M. G. (2017). Nursing care and guidelines for puerperae in rooming-in. Rev Enferm UFPE online. 11(Supl. 9):3508-15. http://dx.doi.org/10.5205/reuol.10620-94529-1-SM.1109sup201702

Paraná. Secretaria de Estado da Saúde do Paraná. (2018). Superintendência de Atenção à Saúde. Linha Guia da Rede Mãe Paranaense. Paraná: Sesa. http://www.saude.pr.gov.br/arquivos/File/LinhaGuiaMae Paranaense_2018.pdf

Pereira, S. B., Diaz, C. M. G., Backes, M. T. S., Ferreira, C. L. L \& Backes, D. S. (2018). Good practices of labor and birth care from the perspective of health professionals. Rev Bras Enferm. 71(Suppl 3):1313-9. http://dx.doi.org/10.1590/0034-7167-2016-0661

Rogerio, M. C., Silva, L., Canario, M. A. S. S., \& Ferrari, R. A. P. (2020). Guidance for puerperas about neonatal care in rooming-in at habitual risk maternities. Enferm Foco. 11 (1): 69-74.

Santos, D. R., Viera, C. S., Guimarães, A. T. B., Toso, B. R. G. O., \& Ferrari, R. A. P. (2020) Assessment of effectiveness of the Rede Mãe Paranaense Program. Saúde Debate. 44(124):70-85. https://doi.org/10.1590/0103-1104202012405

Silva, T. C., Bisognin, P., Prates, L. A., Bortoli, C. F. C., Oliveira, G., \& Ressel, L. B. (2017). Labor and birth care pratices an integrative review. Revista de Enfermagem do Centro-Oeste Mineiro. 7:e 1294. https://doi.org/10.19175/recom.v7i0.1294

Souza, K. V., \& Santos Filho, S. B. (2020). Rede Cegonha network and the methodological challenges of implementing networks in the SUS. Ciênc Saúde Coletiva. 26(3):775-80. https://doi.org/10.1590/1413-81232021263.21462020

Strefling, I. S. S., Borba, C. B., Demori, C. C. \& et al. (2017) Percepções de puérperas sobre o cuidado de enfermagem no alojamento conjunto. Rev Fund Care Online. 9(2):333-339. http://dx.doi.org/10.9789/2175-5361.2017.v9i2.333-339 